\title{
Consensus conference on cyclosporin A microemulsion for psoriasis, June 1996
}

\author{
J.BERTH-JONES AND J.J.VOORHEES* \\ Department of Dermatology. Walsgrave Hospital. Coventry CV2 2DX, U.K. \\ *Department of Dermatology. University of Michigan Medical Center, Ann Arbor, Michigan, U.S.A.
}

\section{Introduction}

Cyclosporin A (CyA) has been registered in over 124 countries world-wide and is used by more than 50,000 psoriasis patients per year. Human studies using oral CyA (Sandimmun ${ }^{(B)}$ ) therapy for psoriasis have been in progress for nearly 13 years, and have confirmed the clinical efficacy of $\mathrm{CyA}$ in this indication. Guidelines have been developed and adopted for the safe use of CyA in psoriasis, ${ }^{1,2}$ and the relative safety of up to 2 years of therapy has been established, provided that the guidelines are observed. However, there is concern regarding the long-term safety of CyA treatment of psoriasis as experience is still limited. Careful monitoring of all CyA-treated patients is therefore mandatory.

This meeting was convened to re-examine the current recommendations for the use of $\mathrm{CyA}$ in psoriasis, in the light of developments since the last consensus conference in $1992 ;^{2}$ namely, the growing understanding of psoriasis, changes in clinical practice (including the use of intermittent short-course therapy), and increased clinical experience with the new microemulsion formulation of CyA (Sandimmun Neoral ${ }^{\circledR}$ ). The principal outcome of the meeting was the creation of an updated set of recommendations for the use of the CyA microemulsion in adult psoriatic patients; these recommendations are presented below. It should be noted, however, that the recommendations are intended only as guidelines and it is assumed that clinical judgement will always be exercised.

\section{Who should be treated?}

CyA is recommended for psoriatic patients requiring systemic treatment, and for whom, in the opinions of both patient and dermatologist, CyA is the most appropriate available option. The psoriasis should be of such severity that the risks inherent in this treatment are justified.

\section{Indications for particular caution in use}

Particular caution and careful clinical judgement are required in patients with: (i) previous or current malignancies (except basal cell carcinoma); (ii) premalignant conditions; (iii) primary or secondary immunodeficiency; (iv) severe hepatic dysfunction; (v) abnormal renal function: (vi) uncontrolled hypertension; (vii) serious infection of any type; (viii) tendency to drug or alcohol abuse; (ix) possible or known pregnancy, and lactation; (x) inability or lack of will to co-operate with regular monitoring.

\section{Drug interactions}

Particular caution is also required in the use of CyA in psoriatic patients who are receiving any additional systemic medication. It is recommended that an up-to-date reference should be consulted regarding possible drug interactions whenever a concomitant medication is used. The following are just some important examples of the numerous treatments with recognized potential to interact with $\mathrm{CyA}$ : (i) alkylating agents such as cyclophosphamide (including prior use); (ii) potentially nephrotoxic agents (e.g. some nonsteroidal anti-inflammatory agents, often prescribed in psoriatic arthritis); (iii) immunosuppressive therapy; (iv) radiation therapy; (v) current ultraviolet (UV) irradiation and previous over-exposure to UV, including both solar irradiation and phototherapy (psoralen and UVA (PUVA) and UVB); (vi) drugs that interfere with the bioavailability or metabolism of cyclosporin, such as anticonvulsants, fluconazole, itraconazole, ketoconazole, erythromycin, trimethoprim, diltiazem and verapamil; (vii) drugs such as diclofenac, prednisolone and lovastatin, the metabolism of which may be affected by cyclosporin.

\section{Recommended procedure prior to therapy}

The patient should be carefully instructed regarding the 
nature and implementation of CyA treatment. Thorough dermatological and physical examinations should be performed, with careful attention being paid to measurement of blood pressure. Serum creatinine should be measured, following a 12-h fast, on at least two separate occasions. If these measurements vary by more than $10 \mu \mathrm{mol} / \mathrm{l}$, further measurements are recommended. The mean value should then be used as the baseline serum creatinine during subsequent monitoring. Assessment of liver enzymes and serum potassium and screening for proteinuria are also recommended. Measurements of bilirubin, magnesium, uric acid and fasting lipids are often performed but are not considered obligatory. The patient should be advised to comply with national recommendations regarding screening procedures for malignancy (e.g. cervical smear, mammogram, palpation of prostate).

\section{Dosage and treatment regimens}

A starting dose of $2.5 \mathrm{mg} / \mathrm{kg}$ body weight per day $(2.5 \mathrm{mg} / \mathrm{kg}$ per day) is recommended for most patients. It is recommended that the total daily dose is divided into two doses (morning and evening), although some dermatologists use a single daily dose. In certain patients where rapid improvement is considered important a higher starting dose may be indicated, but this should not exceed $5 \mathrm{mg} / \mathrm{kg}$ per day.

If improvement in psoriasis has not occurred at 1 month, the CyA dose can be increased gradually, but should not exceed the maximum of $5 \mathrm{mg} / \mathrm{kg}$ per day. The rate of clearance of psoriasis is related to the starting dose. At $2.5 \mathrm{mg} / \mathrm{kg}$ per day, the improvement seen at 1 month is sometimes only modest, but it may nonetheless be appropriate to continue at this dose for up to 3 months before concluding that a higher dose is required. If there is insufficient response after 3 months on the maximum dose of $5 \mathrm{mg} / \mathrm{kg}$ per day, CyA should be discontinued.

When skin lesions improve to an acceptable level, the CyA dose should be reduced in steps of $0.5-1 \mathrm{mg} / \mathrm{kg}$ body weight to the lowest effective dose; if clinical improvement continues, CyA should be discontinued to determine if therapy is still required. Experience indicates that severe psoriasis recurs when $\mathrm{CyA}$ is stopped, as with other therapies. The length of remission appears to be a function of both the severity of the disease and the extent of clearing achieved. Intermittent therapy is preferable whenever possible, with relapse managed by the re-introduction of $\mathrm{CyA}$ at the previous effective dose or by initiating a therapy other than CyA.
In some patients, continuous maintenance therapy may be necessary. The objective of maintenance therapy is not to totally clear the skin, but to maintain substantial clinical improvement with the lowest possible dose of CyA. If a patient experiences serious exacerbation of psoriasis during maintenance therapy, the dose should be increased sufficiently to control the disease. Care should be taken to comply with safety guidelines and the maximum dose should not exceed $5 \mathrm{mg} / \mathrm{kg}$ per day. Every effort should be made to reduce the dose to the lowest effective level.

If patients have been treated successfully for 2 years, the decision whether or not to extend therapy should be based upon the most recent information regarding the long-term use of $\mathrm{CyA}$ in psoriasis. These data continue to accumulate.

\section{Monitoring of patients during treatment}

The use of microemulsified CyA must be carefully monitored and controlled, in the same way as the original formulation. Renal function (i.e. serum creatinine) and blood pressure should be monitored every 2 weeks for the first 6 weeks and monthly thereafter. More frequent monitoring is recommended if the CyA dose is increased or if there is a rise in serum creatinine or blood pressure.

In the event that hypertension (mean diastolic blood pressure $>95 \mathrm{mmHg}$ on two consecutive occasions) develops, the dose of CyA may be reduced or the hypertension may be treated with calcium antagonists such as nifedipine or isradipine. Diltiazem and verapamil are not recommended because they inhibit metabolism of CyA. Potassium-sparing diuretics are probably best avoided as CyA tends to raise serum potassium levels.

If the serum creatinine rises to more than $30 \%$ above the patient's baseline, the measurement should be repeated within 2 weeks. Please note that this 30\% refers to a rise above the patient's own baseline, not to $30 \%$ above the upper limit of normal serum creatinine for the measuring laboratory. If the rise is sustained at this level, the dose of CyA should be reduced by at least $1 \mathrm{mg} / \mathrm{kg}$ per day for at least 1 month. If the creatinine then decreases to less than $30 \%$ above the patient's baseline, treatment can be continued; if not, CyA therapy should be stopped. Treatment should not then be recommenced until the creatinine returns to within $10 \%$ of the patient's baseline. These guidelines are summarized in the algorithm in Fig. 1.

If the serum creatinine repeatedly rises to more than 


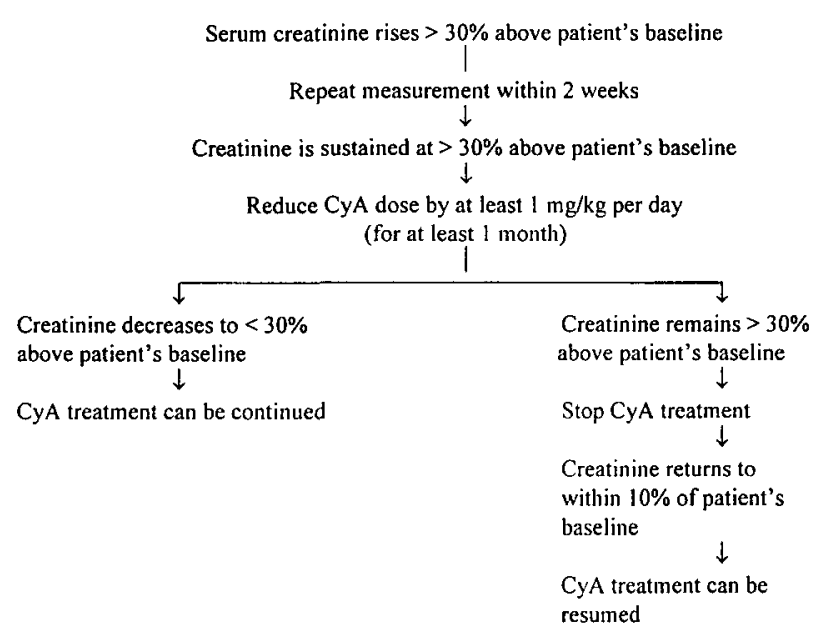

Figure 1. Steps to follow with rising creatinine.

$30 \%$ above the patient's baseline when CyA is re-introduced, then either the CyA should be discontinued and an alternative treatment adopted for the psoriasis, or advice should be obtained from a nephrologist. Extreme caution is also required if the serum creatinine is within $30 \%$ of baseline for an individual but lies outside the normal range, as this may be indicative of pre-existing renal impairment.

Other side-effects, such as tremor, hypertrichosis, gingival hyperplasia or nausea, should be recorded. If these cause concern, the dose should be reduced in steps or, if necessary, CyA should be discontinued.

\section{Conversion from the original formulation to the microemulsion formulation}

When converting patients from the original CyA formulation to the microemulsion formulation, a $1: 1$ dose-conversion strategy is recommended. In the majority of patients who absorb the original formulation adequately, the absorption of $\mathrm{CyA}$ is unlikely to change post-conversion. However, in patients who were relatively poor absorbers of $\mathrm{CyA}$ from the original formulation, absorption will increase post-conversion. As a result, it may be necessary to make subsequent dose-reductions in these patients to ensure that they are receiving the lowest effective dose. Dose adjustments should also be made, as required, to comply with the safety guidelines above.

Careful safety monitoring is mandatory postconversion. Blood pressure and serum creatinine should be measured prior to conversion and 2, 4 and 8 weeks thereafter. Hypertension or an increase in serum creatinine should be managed according to the guidelines above.

\section{Participation and organization of consensus}

This consensus took place in Rome, Italy, on 23 June 1996, under the sponsorship of Sandoz Pharma Ltd (Basel, Switzerland). The co-chairmen were Dr J.BerthJones, Department of Dermatology, Walsgrave Hospital, Coventry, U.K., and Prof. J.J.Voorhees, Department of Dermatology, University of Michigan, Ann Arbor, Michigan, U.S.A.

Other participants were Dr B.Abrams, Sandoz Research Institute, East Hanover, New Jersey, U.S.A.; Dr B.R.Allen, Department of Dermatology, University Hospital, Queen's Medical Centre, Nottingham U.K.; Prof. J.D.Bos, Department of Dermatology, University of Amsterdam. Academisch Medisch Centrum, Amsterdam, The Netherlands; Prof. E.Christophers, Universitätsklinik, Kiel, Germany; Prof. A.F.Finzi, Institute of Dermatology, University of Milan, Milan, Italy; Dr W.Gulliver, Memorial University of Newfoundland, St John's, Canada; Dr W.Harrison, Sandoz Pharma Ltd, Basel, Switzerland; Dr V.C.Ho, Division of Dermatology, University of British Columbia, Vancouver, Canada; Dr J.Koo, UCSF Psoriasis Treatment Center, University of California, San Francisco Medical Center, San Francisco, California, U.S.A.; Dr A.-G.Schmidt, Sandoz Pharma Ltd, Basel, Switzerland; Dr A.Stütz, Sandoz Forschungsinstitut, Vienna, Austria.

The authors wish to acknowledge the contribution of Prof. M.J.Mihatsch in reviewing this report.

\section{References}

1 A consensus report: cyclosporin A therapy for psoriasis. $\mathrm{Br}$ J Dermatol 1990; 122 (Suppl. 36): 1-3.

2 Mihatsch MJ, Wolff K. Consensus conference on cyclosporin A for psoriasis. February 1992. Br J Dermatol 1992; 126: 621-3. 
This document is a scanned copy of a printed document. No warranty is given about the accuracy of the copy. Users should refer to the original published version of the material. 